OPEN

SUBJECT AREAS:

TERAHERTZ OPTICS

METAMATERIALS

ULTRAFAST PHOTONICS

NANOPHOTONICS AND

PLASMONICS

Received

16 September 2014

Accepted

31 December 2014

Published

27 January 2015

Correspondence and requests for materials should be addressed to T.F. (thomas.feurer@ iap.unibe.ch) or S.B. (salvatore.bagiante@ psi.ch)

\section{Giant Electric Field Enhancement in Split Ring Resonators Featuring Nanometer-Sized Gaps}

\author{
S. Bagiante ${ }^{1,2}$, F. Enderli ${ }^{1,2}$, J. Fabiańska ${ }^{2}$, H. Sigg' \& T. Feurer ${ }^{2}$ \\ 'Laboratory of Micro- and Nanotechnology Paul Scherrer Institute, Villigen 5232, Switzerland, ${ }^{2}$ Institute of Applied Physics \\ University of Bern, Bern 3012, Sidlerstrasse 5, Switzerland.
}

Today's pulsed THz sources enable us to excite, probe, and coherently control the vibrational or rotational dynamics of organic and inorganic materials on ultrafast time scales. Driven by standard laser sources $\mathrm{THz}$ electric field strengths of up to several $\mathrm{MVm}^{-1}$ have been reported and in order to reach even higher electric field strengths the use of dedicated electric field enhancement structures has been proposed. Here, we demonstrate resonant electric field enhancement structures, which concentrate the incident electric field in sub-diffraction size volumes and show an electric field enhancement as high as $\sim 14,000$ at $50 \mathrm{GHz}$. These values have been confirmed through a combination of near-field imaging experiments and electromagnetic simulations.

( ver the past 20 years, continuous progress in Terahertz $(\mathrm{THz})$ technologies has facilitated numerous breakthroughs in scientific and industrial research ${ }^{1-3}$. Mostly through linear time-domain $\mathrm{THz}$ spectroscopy we have witnessed a marked increase in THz research on molecules ${ }^{4}$, biomolecules ${ }^{5}$, liquids ${ }^{6}$, semiconductors ${ }^{5}$, superconductors ${ }^{7}$, crystals or complex materials ${ }^{8}$. Moreover, THz spectroscopy forms the basis for stand-off detection of hidden chemicals ${ }^{9,10}$; in comparison to visible or infrared radiation, THz frequencies can penetrate into organic materials such as skin, plastics, cloths, or paper products and have thus become indispensable in security applications. An additional benefit is that $\mathrm{THz}$ radiation, in contrast to $\mathrm{X}$-rays, does not cause any damage associated with ionization ${ }^{11}$.

Despite all progress, the generation of $\mathrm{THz}$ pulses with high peak electric fields is still rather limited, mostly due to technological constraints such as available laser power and low conversion efficiencies ${ }^{5,12}$. The highest $\mathrm{THz}$ powers are currently available from large-scale electron particle accelerators ${ }^{13}$, but also tabletop sources show similar powers when using, for example, large area photoconductive switches, frequency mixing in laser generated plasmas, or optical rectification in nonlinear crystals $^{14-18}$. In order to extend $\mathrm{THz}$ experiments into the nonlinear regime the quantity to be optimized is the electric field strength $E^{19}$. It is linked to the $\mathrm{THz}$ pulse energy $Q$ through the relation $E \propto \sqrt{Q / \Delta \tau A}$ where $\Delta \tau$ is the temporal pulse duration and $A$ the beam area. The available pulse energy is naturally limited by the THz system at hand and the pulses are often already single-cycle, so that the electric field strength cannot be increased by adjusting the parameters $Q$ and $\Delta \tau$. The beam area can be minimized through tight focusing, however, the diffraction limit imposes a lower boundary on $A$.

Diffraction limited focusing can be overcome, for example, by using suitable metallic structures which act as antennas efficiently collecting the incident radiation and concentrating it in a small sub-wavelength sized volume. In the process, part of the incident radiation is typically converted to charge density oscillations, which in turn generate an enhanced and localized electric field distribution on a length scale well beyond the diffraction limit ${ }^{20-23}$. At visible wavelengths this concept has turned into a key element for example for single-molecule spectroscopy, nano-imaging, or extreme nonlinear optics ${ }^{24-26}$. Recently, Seo et al. ${ }^{21}$ and Blanchard et al. ${ }^{22}$ have demonstrated that similar concepts are applicable in the $\mathrm{THz}$ regime. By illuminating a dipole antenna in form of a nano-slit Seo and coworkers demonstrated strong electric field localization. For a $70 \mathrm{~nm}$ wide nano-slit and a frequency of $0.1 \mathrm{THz}$ the electric field enhancement was on the order of $1,000^{21,27}$.

Here, we report on a novel antenna design, i.e. a split ring resonator featuring a nanometer sized gap, which extends into the inner part of the split ring resonator. Such structures, as outlined below, show promise for extremely high $\mathrm{THz}$ electric field enhancement at their resonance frequencies. These frequencies can be easily tuned throughout the entire $\mathrm{THz}$ spectrum by changing the structure's dimensions. Since there exists no detec- 
tion modality with sufficient spatial resolution to map out the in-gap electric field we have to resort to indirect methods. Based on nearfield measurements of the oscillating charge distribution on the split ring resonator we calibrate detailed finite element simulations to derive the $\mathrm{THz}$ electric field enhancement and $\mathrm{THz}$ electric field amplitude in the gap. Throughout the manuscript the electric field enhancement as well as the in-gap electric field are averaged over the gap volume and may be lower or higher at specific points within the gap. At the lowest order resonance of the specific design we find that the $\mathrm{THz}$ electric field enhancement reaches 14,300 at $50 \mathrm{GHz}$ for a $100 \mathrm{~nm}$ wide gap and is the highest reported to date in this frequency regime. The corresponding maximum $|E|^{2}$ and $|E|^{4}$ enhancement factors are $2 \cdot 10^{8}$ and $4 \cdot 10^{16}$, respectively, exceeding values typically observed in surface enhanced Raman spectroscopy ${ }^{28}$. Therefore, such antenna structures are an excellent platform for nonlinear $\mathrm{THz}$ science, small $\mathrm{THz}$ signal detection, or few molecule and nano-particle detection. By irradiating the structures with $\mathrm{THz}$ pulses from a standard femtosecond oscillator driven spectroscopy source we find in-gap electric field strengths of approximately $80 \mathrm{kVm}^{-1}$; such electric field strength are usually only achieved with amplified femtosecond laser systems.

\section{Results}

Figure 1a) shows an image of a split ring resonator with extended capacitive faces and a close-up view of the gap recorded with a scanning electron microscope. The length of the extended capacitive faces can be adjusted to control the gap volume and therefore the interaction volume without changing the area covered by the split ring resonator ${ }^{29,30}$. The substrate is high resistivity silicon and the relevant dimensions of the gold structures investigated hereafter are $L=200 \mu \mathrm{m}, w=10 \mu \mathrm{m}, s=20 \mu \mathrm{m}$, and $h=60 \mathrm{~nm}$ (see Figure 1b). Three different isolated split ring resonators were fabricated featuring gap widths of $g=100 \mathrm{~nm}, 500 \mathrm{~nm}$, and $970 \mathrm{~nm}$, respectively. The total length of the unfolded ring was chosen such as to find the fundamental and the third resonance at approximately $v_{1}=60 \mathrm{GHz}$ and $v_{3}=180 \mathrm{GHz}$. Note that even order resonances are not excited here. For these parameters the structure size is well matched to the $20 \mu \mathrm{m}$ spatial resolution of the $\mathrm{THz}$ near-field imaging setup ${ }^{31-33}$.

At the fundamental resonance, the incident $\mathrm{THz}$ radiation induces a current flow on the metal surface that leads to an accumulation of charge carriers around the gap region. This capacitive charging in turn results in an in-gap electric field enhancement by orders of magnitude. The electromagnetic energy stored in the SRR and, thus, the amount of charge carriers accumulating in the gap region is finite. For increasing gap length, the charge accumulations are therefore distributed over a larger distance, so that the field enhancement is found to decrease $\mathrm{e}^{29}$. For comparison, the magnetic field enhancement is typically one to two orders of magnitude smaller than the
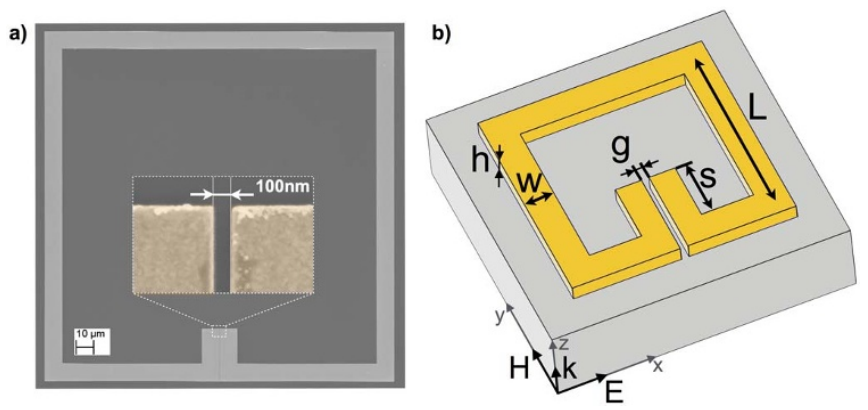

Figure $1 \mid$ (a) SEM image of the split ring resonator and a close-up view, which shows part of the $100 \mathrm{~nm}$ wide gap region. (b) Schematic

illustration of the split ring resonator with all relevant dimensions, the $(E$, $H, k)$ triad of the incident $\mathrm{THz}$ field, $k$ refers to the wave vector, and the coordinate system $(x, y, z)$. electric field enhancement. At higher order resonances, the induced current flow shows a more complex pattern and typically results in somewhat lower electric field enhancement values ${ }^{34}$. Since no $\mathrm{THz}$ detection system has the spatial resolution required to directly measure, let alone map out, the electric field distribution in the gap volume, we resort to quantify the charge distribution in the split ring resonator, which is responsible for the electric field enhancement in the gap. For this we use a $\mathrm{THz}$ near-field setup (for more experimental details see also supplementary information) configured to measure the electric field component normal to the plane containing the split ring resonator, which is directly proportional to the charge distribution.

Exemplarily, we discuss the results for the split ring resonator with a $500 \mathrm{~nm}$ wide gap. The incident single-cycle $\mathrm{THz}$ pulses cover a spectral range from $10 \mathrm{GHz}$ to about $1.5 \mathrm{THz}$ with the peak at approximately $0.32 \mathrm{THz}$. They propagate along the $z$-axis and the sample is positioned at the image plane of the THz source. There, the $\mathrm{THz}$ electric field is polarized primarily along the $x$-axis and is parallel to the side of the split ring resonator which contains the gap. The $(E, H, k)$ triad - where $E$ is the electric and $H$ the magnetic field and $k$ the wave vector - of the incident THz pulse is indicated in Fig. 1b). Figure 2e) shows the measured time dependent out-of-plane electric field $E_{z}(t)$ averaged over 9 pixels around the top right corner of the split ring resonator. Note that the incident $\mathrm{THz}$ pulse has a negligible longitudinal electric field component and the measured electric field is solely due to the $\mathrm{THz}$-induced charge oscillations. Figures 2a) to d) show snapshots of the measured out-of-plane electric field distribution $E_{z}\left(x, y, t_{j}\right)$ at successive times $t_{j}=9.2 \mathrm{ps}, 10 \mathrm{ps}, 10.8 \mathrm{ps}$, and $11.8 \mathrm{ps}$ as marked by the vertical red lines in Fig. 2e). The color scale indicates the absolutely calibrated electric field (see supplementary information).

From the calibrated temporal electric field distribution $E_{z}(x, y, t)$ we calculate the spectral electric field distribution $E_{z}(x, y, v)$ by Fourier transforming the time dependent electric field at every position $(x, y)$. The intensity graph in Fig. 3a) shows the measured electric field distribution $\left|E_{z}\left(x, y, v_{3}\right)\right|$ at the third resonance, i.e. at $v_{3}=$ $180 \mathrm{GHz}$. The signal at the first resonance is close to the noise floor and not considered here.

Comparing the measured (Fig. 3a) to the simulated (Fig. 3b) spatial distribution of the out-of-plane electric field $\left|E_{z}\left(x, y, v_{3}\right)\right|$ shows excellent agreement. The simulations are performed in frequencydomain and in three spatial dimensions using a commercial software package (COMSOL Multiphysics) ${ }^{35}$. The simulations take into account the substrate on which the split ring resonator structure is fabricated as well as the electro-optic detection crystal, which is an integral part of the near-field detection system. The only free parameter in the simulations is the electric field strength of the incident $\mathrm{THz}$ radiation, which we determine from a separate measurement of $E_{x}(t)$ with no split ring resonator on the substrate. The simulations not only yield the out-of-plane electric field distribution but any other relevant quantity, most importantly the electric field distribution and the electric field enhancement in the gap where the measurements, due to the limited spatial resolution, cannot reveal any information. That is, we experimentally verify that the simulations accurately reproduce the spatially resolved out-of-plane electric field at the third resonance $\left(v_{3}=180 \mathrm{GHz}\right)$, and thus the charge distribution on the split ring resonator which is responsible for the capacitive charging of the gap, and then extract the in-gap electric field and field enhancement from the simulations.

Figure 4a) shows the simulated in-gap electric field enhancement as a function of frequency for all three gap widths examined. The black vertical dashed line marks the calibration points for all three gap widths at the third order resonance frequencies $v_{3}$. For a $100 \mathrm{~nm}$ wide gap the maximum electric field enhancement is as high as 14300 at the fundamental resonance and 3400 at the third order resonance. As mentioned above the electric field enhancement at the individual 

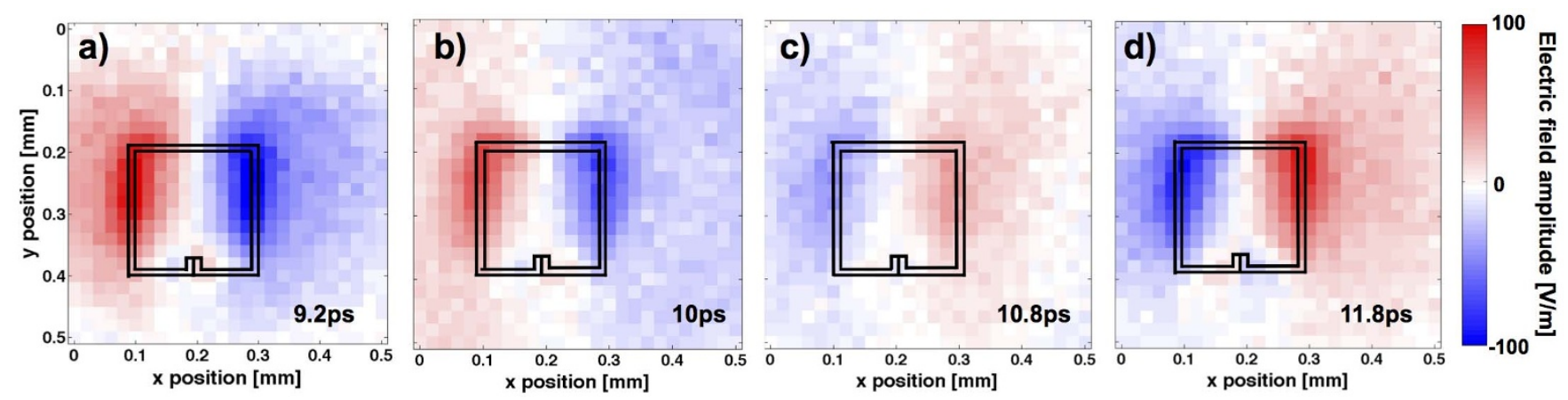

a b

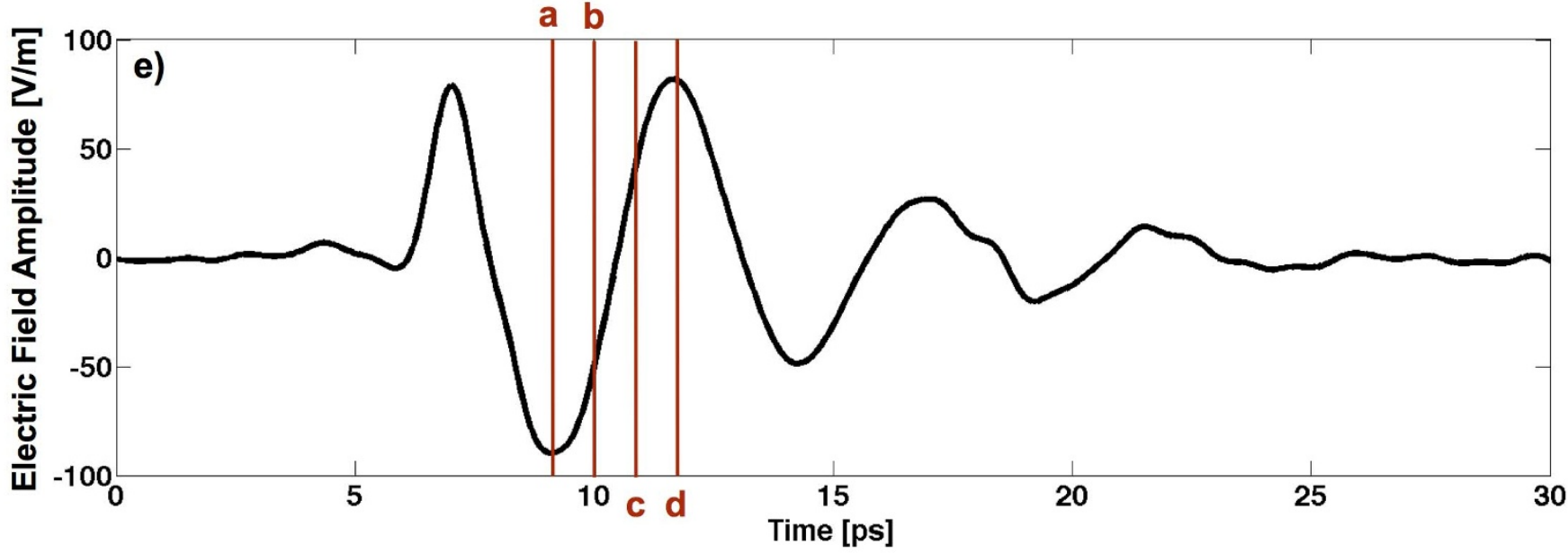

Figure $2 \mid$ Measured time dependent out-of-plane electric field for a split ring resonator with a $500 \mathbf{n m}$ wide gap. The intensity graphs show the electric field distribution $E_{z}\left(x, y, t_{j}\right)$ at successive times $t_{j}=9.2 \mathrm{ps}(\mathrm{a}), 10 \mathrm{ps}(\mathrm{b}), 10.8 \mathrm{ps}(\mathrm{c})$, and $11.8 \mathrm{ps}(\mathrm{d})$, respectively. The time dependent electric field measured at the top right corner of the structure is shown in e) and the $t_{j}$ are marked by red vertical lines. The supplementary online material contains a movie showing the full time dependent electric field distribution $E_{z}(x, y, t)$ from which Figs. (a)-(d) were extracted.

resonances differ because of the different character of the charge distribution on the split ring structure. At the lowest order resonance the charge is known to accumulate predominantly around the gap. Conversely, at the third order resonance, the charges accumulate at the two corners opposite to the gap ${ }^{36}$, therefore the electric field enhancement is lower. The spatial in-gap distribution of the electric field enhancement in the xy plane (at $\mathrm{z}=\mathrm{h} / 2=30 \mathrm{~nm}, \mathrm{~g}=500 \mathrm{~nm}$, $v_{1}=56 \mathrm{GHz}$ ) is shown in Figure $4 \mathrm{~b}$ ). Except some variations at the edges, it is relatively homogeneous in the entire region.

\section{Electric field amplitude $[\mathrm{V} / \mathrm{m}]$}

0
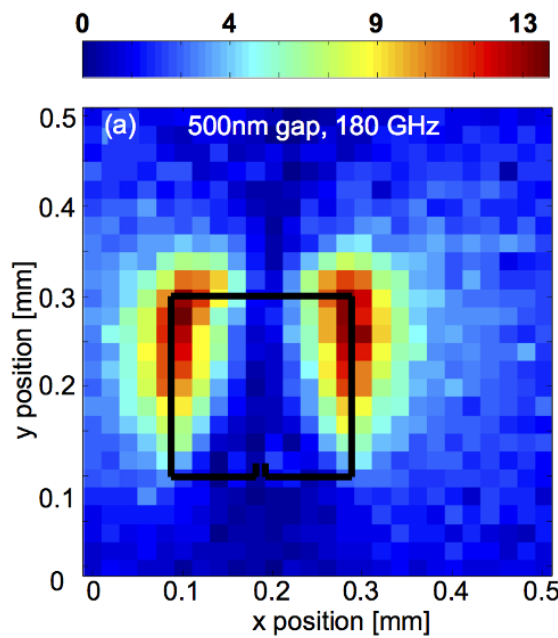

Figure 4c) shows the calibrated in-gap electric field as obtained by multiplying the incident $\mathrm{THz}$ spectrum (gray shaded area in Fig. 4a)) with the complex-valued electric field enhancement for the $100 \mathrm{~nm}$ wide gap. The phase associated to the absolute value of the electric field enhancement shown in Fig. 4a) was calculated by means of Kramer-Kronig's relation. The in-gap $\mathrm{THz}$ electric field has two distinct frequency components, i.e. a short lived at $v_{3}=175 \mathrm{GHz}$ (broad line width) and a long lived at $v_{3}=50 \mathrm{GHz}$ (narrow line width), and reaches peak values on the order of $80 \mathrm{kVm}^{-1}$. The
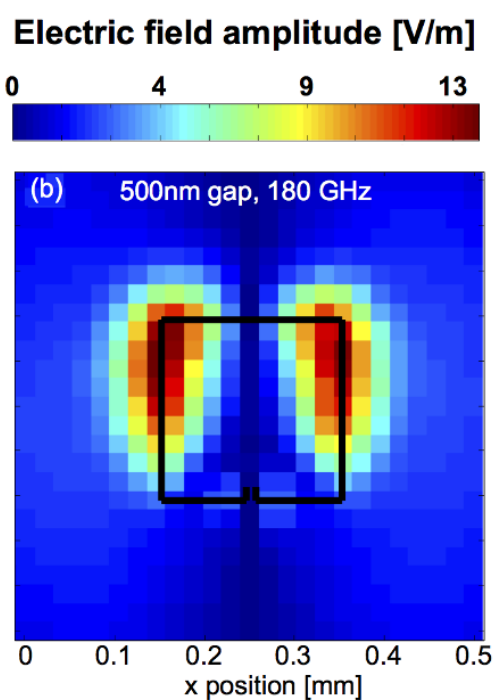

Figure $3 \mid$ Intensity graphs of the measured a) and the simulated b) out-of-plane electric field distribution $\left|E_{z}\left(x, y, v_{3}\right)\right|$ at the third resonance. 

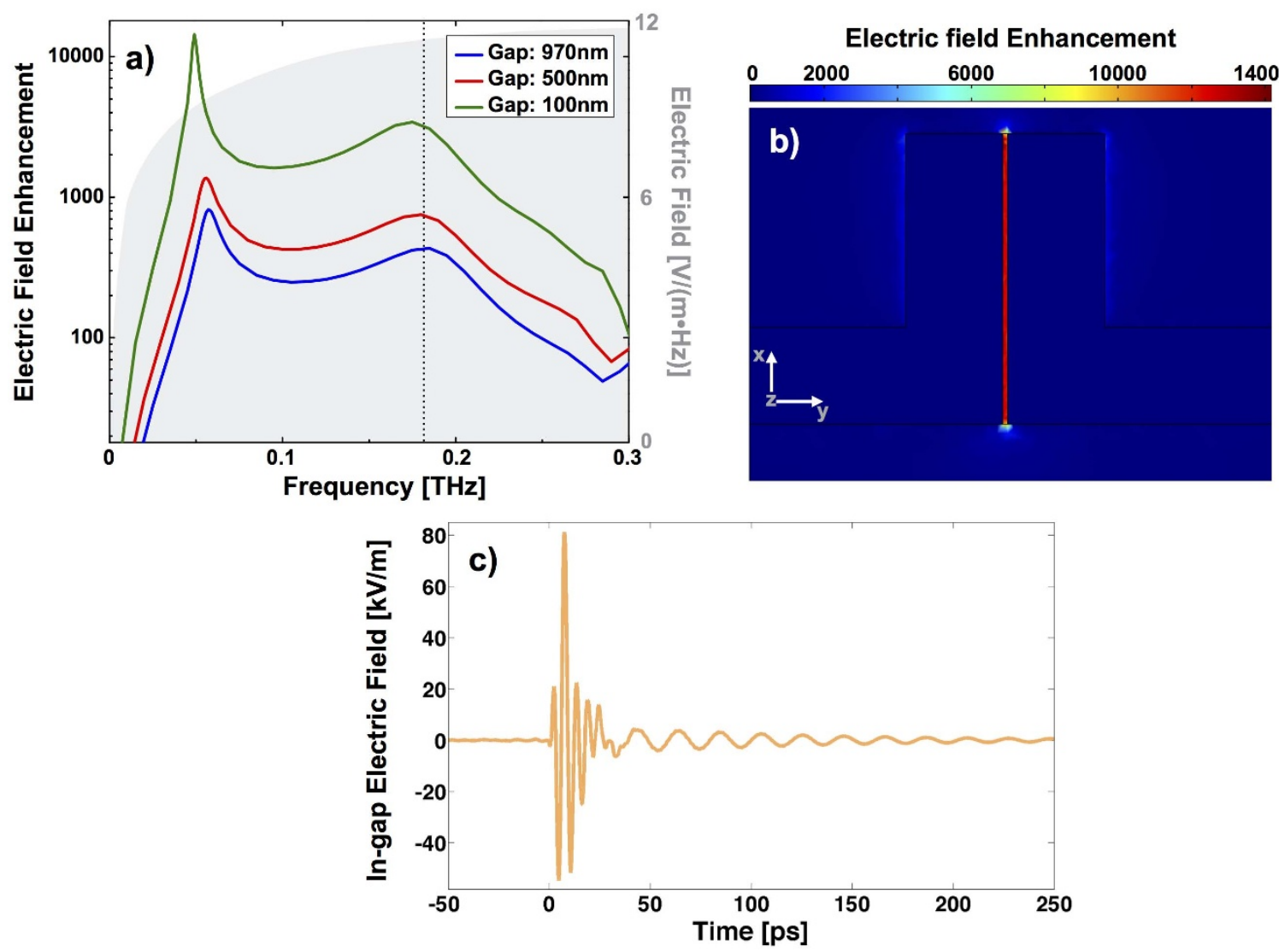

Figure $4 \mid$ (a) Absolute value of the electric field enhancement in the gap as a function of frequency for the three gap widths $100 \mathrm{~nm}$, $500 \mathrm{~nm}$, and $970 \mathrm{~nm}$, respectively. Absolute electric field amplitude of the incident THz spectrum in gray. (b) Electric field enhancement distribution in a $500 \mathrm{~nm}$ gap SRR at $56 \mathrm{GHz}$. (c) In-gap electric field strength versus time for the $100 \mathrm{~nm}$ wide gap.

results suggest that even a conventional tabletop $\mathrm{THz}$ source, as it is used in many $\mathrm{THz}$ time domain spectrometers, combined with such a split ring resonator can result in electric field amplitudes as high as several hundreds of $\mathrm{kVm}^{-1}$. A more powerful $\mathrm{THz}$ source will yield electric fields sufficiently high for many nonlinear $\mathrm{THz}$ effects, for example in semiconductors.

We would like to point out that the dimensions of the split ring resonators used here have been chosen to allow for sophisticated near-field $\mathrm{THz}$ imaging and not for achieving the highest possible electric field strengths. For such purpose one would aim for a better overlap between the incident $\mathrm{THz}$ spectrum and the electric field enhancement curve. Although available fabrication techniques permit narrower gap widths (the smallest gap width is most likely limited by the Thomas-Fermi screening length when non-local effects have to be considered; this occurs below $1 \mathrm{~nm}$ ), and thus even higher electric field enhancements, these are not necessarily advantageous as they result in a smaller and more difficult to access interaction volume. Therefore, a compromise should be found between electric field enhancement and interaction volume.

\section{Discussion}

Through a combination of $\mathrm{THz}$ near-field imaging measurements and sophisticated electromagnetic simulations we have demonstrated giant electric field enhancement in split ring resonators featuring a nano-gap. Electric field enhancement values as high as $\sim 14,000$ and $\sim 3,400$ were observed for a $100 \mathrm{~nm}$ wide gap with the resonance frequencies at $50 \mathrm{GHz}$ and $175 \mathrm{GHz}$. At $50 \mathrm{GHz}$ the corresponding maximum $|E|^{2}$ and $|E|^{4}$ enhancement factors are $2 \cdot 10^{8}$ and $4 \cdot 10^{16}$. Through a judicious choice of the split ring resonator's dimensions the resonances can be easily tuned throughout the entire
$\mathrm{THz}$ region, and thus matched to vibrational or rotational resonances in molecules or phonon resonances in solids. Moreover, their width can be adjusted by exploiting strong couplings to other resonant structures as shown for example in reference ${ }^{37}$. These results represent an important step towards sub-diffraction limited $\mathrm{THz}$ focusing, $\mathrm{THz}$ electric field enhancement, and their use in linear and nonlinear $\mathrm{THz}$ experiments. Using appropriate resonators, nonlinear experiments starting from even common laboratory $\mathrm{THz}$ sources are conceivable.

1. Tonouchi, M. Cutting-edge terahertz technology. Nat. Photonics 1, 97-105 (2007).

2. Liua, Y. \& Zhang, X. Metamaterials: a new frontier of science and technology. Chem. Soc. Rev 40, 2494-2507 (2011).

3. Blanchard, F., Doi, A., Tanaka, T. \& Tanaka, K. Real-Time, Subwavelength Terahertz Imaging. Annu. Rev. Mater. Res. 43, 237-259 (2013).

4. Park, H. R. et al. Colossal Absorption of Molecules Inside Single Terahertz Nanoantennas. Nano Lett. 13, 1782-1786 (2013).

5. Ferguson, B. \& Zhang, X. C. Materials for terahertz science and technology. Nat. Mater. 1, 26-33 (2002).

6. Rønne, C., Åstrand, P. O. \& and Keiding, S. R. THz Spectroscopy of Liquid $\mathrm{H}_{2} \mathrm{O}$ and $\mathrm{D}_{2} \mathrm{O}$. Phys. Rev. Lett. 82, 2888-2891 (1999).

7. Ozyuzer, L. et al. Emission of Coherent THz Radiation from Superconductors. Science 318, 1291-1293 (2007).

8. Slepyan, G. Ya, Shuba, M. V., Maksimenko, S. A., Thomsen, C. \& Lakhtakia, A. Terahertz conductivity peak in composite materials containing carbon nanotubes: Theory and interpretation of experiment. Phys. Rev. B 81, 205423 (2010).

9. Shen, Y. C. et al. Detection and identification of explosives using terahertz pulsed spectroscopic imaging. Appl. Phys. Lett. 86, 241116 (2005).

10. Louise, H., Pepper, M. \& Taday, P. Terahertz spectroscopy: Signatures and fingerprints. Nat. Photonics 2, 541-543 (2008).

11. Kawase, K., Ogawa, Y. \& Watanabe, Y. Non-destructive terahertz imaging of illicit drugs using spectral fingerprints. Opt. Express 20, 2549-2554 (2003). 
12. Razzari, L. et al. Nonlinear ultrafast modulation of the optical absorption of intense few-cycle terahertz pulses in n-doped semiconductors. Phys. Rev. B 79, 193204 (2009)

13. Casalbuoni, S., Schmidt, B., Schmüser, P., Arsov, V. \& Wesch, S. Observation of Gigawatt-Class $\mathrm{THz}$ Pulses from a Compact Laser-Driven Particle Accelerator. Phys. Rev. ST Accel. Beams 12, 030705 (2009).

14. Jones, R. R., You, D. \& Bucksbaum, P. H. Ionization of Rydberg atoms by subpicosecond half-cycle electromagnetic pulses. Phys. Rev. Lett. 70, 1236-1239 (1993).

15. Bartel, T., Gaal, P., Reimann, K., Woerner, M. \& Elsaesser, T. Generation of singlecycle THz transients with high electric-field amplitudes. Opt. Lett. 30, 2805-2807 (2005).

16. Kim, K.-Y., Glownia, J. H., Taylor, A. J. \& Rodriguez, G. Terahertz emission from ultrafast ionizing air in symmetry-broken laser fields. Opt. Express 15, 4577-4584 (2007).

17. Bass, M., Franken, P. A., Ward, J. F. \& Weinreich, G. Optical rectification. Phys. Rev. Lett. 9, 446-448 (1962).

18. Hebling, J. N., Yeh, K.-L., Hoffmann, M. C. \& Nelson, K. A. High-Power THz Generation, THz Nonlinear Optics, and THz Nonlinear Spectroscopy. IEEE J. Sel. Top. Quant. Electr. 14, 345-353 (2008).

19. Zhang, X.-C. Bright terahertz sources. Nat. Photonics 7, 670-671 (2013)

20. Ebbesen, T., Lezec, H., Ghaemi, H., Thio, T. \& Wolff, P. Extraordinary optical transmission through sub-wavelength hole arrays. Nature 391, 667-669 (1998).

21. Seo, M. A. et al. Terahertz field enhancement by a metallic nano slit operating beyond the skin-depth limit. Nat. Photonics 3, 152-156 (2009).

22. Blanchard, F., Ooi, K., Tanaka, T., Doi, A. \& Tanaka, K. Terahertz spectroscopy of the reactive and radiative near-field zones of split ring resonator. Opt. Express 20, 19395-19403 (2012).

23. Feuillet-Palma, C., Todorov, Y., Vasanelli, A. \& Sirtori, C. Strong near field enhancement in THz nano-antenna arrays. Sci. Rep. 3, 1-8 (2013).

24. Bharadwaj, P., Beams, R. \& Novotny, L. Nanoscale spectroscopy with optical antennas. Chem. Sci. 2, 136-140 (2011).

25. De Angelis, F. et al. Nanoscale chemical mapping using three-dimensional adiabatic compression of surface plasmon polaritons. Nat. Nanotech. 5, 67-72 (2010).

26. Kim, S. et al. High-harmonic generation by resonant plasmon field enhancement. Nature 453, 757-760 (2008).

27. Shalaby, M. et al. Concurrent field enhancement and high transmission of $\mathrm{THz}$ radiation in nanoslit arrays. Appl. Phys. Lett. 99, 041110 (2011).

28. Stiles, P. L., Dieringer, J. A., Shah, N. C. \& Van Duyne, R. P. Surface-Enhanced Raman Spectroscopy. Annu. Rev. Anal. Chem. 601-626 (2008).

29. Merbold, H., Bitzer, A. \& Feurer, T. Second harmonic generation based on strong field enhancement in nanostructured THz materials. Opt. Express 19, 7262-7273 (2011).

30. Schurig, D. et al. Metamaterial electromagnetic cloak at microwave frequencies. Science 314, 977-980 (2006).
31. Hu, B. \& Nuss, M. Imaging with terahertz waves. Opt. Lett. 20, 1716-1718 (1995).

32. Tsai, H.-R., Enderli, F., Feurer, T. \& Webb, K. J. Optimization-Based Terahertz Imaging. IEEE Trans. THz Sci. Tech. 2, 493-503 (2012).

33. Zhu, W. \& Nahata, A. Electric field vector characterization of terahertz surface plasmons. Opt. Express 15, 5616-5624 (2007).

34. Bitzer, A. et al. Terahertz near-field imaging of electric and magnetic resonances of a planar metamaterial. Opt. Express. 17, 3826-3834 (2009).

35. Littmarck, S. \&Saeidi, F. Comsol Multiphysics (1986)Available at: http://www. comsol.com. (Accessed: 11th November 2011).

36. Rockstuhl, C. et al. On the interpretation of resonances in split-ring-resonators at normal incidence. Opt. Express. 14, 8827-8836 (2006).

37. Merbold, H., Bitzer, A. \& Feurer, T. Near-field investigation of induced transparency in similarly oriented double split-ring resonators. Opt. Lett. 36, 1683-1685 (2011).

\section{Acknowledgments}

This work was supported by the Swiss National Research Foundation (SNSF) through the NCCR-MUST and through project 200020-140943. S. Bagiante acknowledges financial support by the Marie Curie COFUND program.

\section{Author contributions}

F.E. and S.B. performed the experiments; S.B. fabricated the sample; J.F. and F.E. performed the simulations; S.B., H.S. and T.F. wrote the manuscript; all authors discussed the results and contributed to the manuscript.

\section{Additional information}

Supplementary information accompanies this paper at http://www.nature.com/ scientificreports

Competing financial interests: The authors declare no competing financial interests.

How to cite this article: Bagiante, S., Enderli, F., Fabiańska, J., Sigg, H. \& Feurer, T. Giant Electric Field Enhancement in Split Ring Resonators Featuring Nanometer-Sized Gaps. Sci. Rep. 5, 8051; DOI:10.1038/srep08051 (2015)

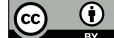

This work is licensed under a Creative Commons Attribution 4.0 International License. The images or other third party material in this article are included in the article's Creative Commons license, unless indicated otherwise in the credit line; if the material is not included under the Creative Commons license, users will need to obtain permission from the license holder in order to reproduce the material. To view a copy of this license, visit http://creativecommons.org/licenses/by/4.0/ 